\title{
The role of carbon-ion radiotherapy in the treatment of adenoid cystic carcinoma of the nasopharynx
}

\author{
Jiyi Hu ${ }^{1,2 \#}$, Weixu Hu${ }^{1,2 \#}$, Jing Gao ${ }^{1,2}$, Jing Yang ${ }^{1,2}$, Xianxin Qiu ${ }^{1,2}$, Qingting Huang ${ }^{1,2}$, Lin Kong ${ }^{2,3}$, \\ Jiade J. Lu ${ }^{1,2}$
}

${ }^{1}$ Department of Radiation Oncology, Shanghai Proton and Heavy Ion Center, Shanghai, China; ${ }^{2}$ Shanghai Engineering Research Center of Proton and Heavy Ion Radiation Therapy, Shanghai, China; ${ }^{3}$ Department of Radiation Oncology, Shanghai Proton and Heavy Ion Center, Fudan University Cancer Center, Shanghai, China

Contributions: (I) Conception and design: J Hu, L Kong, JJ Lu; (II) Administrative support: L Kong, JJ Lu; (III) Provision of study materials or patients: None; (IV) Collection and assembly of data: J Hu, W Hu, J Gao, J Yang, X Qiu; (V) Data analysis and interpretation: J Hu, W Hu, L Kong, JJ Lu; (VI) Manuscript writing: All authors; (VII) Final approval of manuscript: All authors.

\#These authors contributed equally to this work.

Correspondence to: Lin Kong; Jiade J. Lu. Shanghai Proton and Heavy Ion Center, 4365 Kangxin Road, Pudong, Shanghai 201321, China.

Email: lin.kong@sphic.org.cn; jiade.lu@sphic.org.cn.

Background: Nasopharyngeal adenoid cystic carcinoma (NACC) is a distinct subgroup of adenoid cystic carcinoma (ACC) with limited surgical access but predilection of regional and distant metastasis. Although radiotherapy is an integral treatment for patients with NACC, photon-based radiotherapy yielded suboptimal local control. Because of its advantages in biology and physics properties, carbon-ion radiotherapy (CIRT) was attempted for the treatment of head and neck ACC; however, the use of CIRT specifically for NACC has not been investigated.

Methods: Patients with NACC that received CIRT alone or a combination of CIRT and proton beam therapy (PBT) at the Shanghai Proton and Heavy Ion Center (SPHIC) between July 2016 and March 2019 were included in the analysis. Patients with newly diagnosed NACC received combined therapy of CIRT (as boost) and PBT, and those with recurrent disease received CIRT alone. Overall survival (OS), local progression-free survival (LPFS), regional progression-free survival (RPFS), and distant metastasis-free survival (DMFS) were calculated by Kaplan-Meier method.

Results: A total of 22 patients were included in this analysis. Among those, 18 patients had newly diagnosed NACC (17 with locally advanced disease), and 4 had recurrent NACC including 2 failed previous irradiation. After a median follow-up of 30.9 months, the 2-year OS rate, PFS rate, LPFS rate, RPFS rate and DMFS rate were $100 \%, 84.8 \%, 94.4 \%, 100 \%$, and $84.8 \%$, respectively. Three patients experienced grade 3 mucositis or xerostomia. No late toxicity of grade $\geq 3$ was observed.

Conclusions: CIRT alone or in combination with PBT appeared to be a promising modality for the treatment of NACC and produced satisfactory local disease control and toxicity profile. Distant metastasis remained to be a substantial mode for treatment failure. Further follow-up is necessary to evaluate long-term survivals and late toxicity profile.

Keywords: Nasopharyngeal adenoid cystic carcinoma (NACC); carbon-ion radiotherapy (CIRT); treatment outcome; toxicities

Submitted Feb 20, 2020. Accepted for publication Apr 07, 2020.

doi: 10.21037/atm-20-1767

View this article at: https://dx.doi.org/10.21037/atm-20-1767 


\section{Introduction}

Adenoid cystic carcinoma (ACC) is a relatively rare salivary gland tumor accounting for about $1 \%$ of the head and neck malignancies. Nasopharyngeal ACC (NACC) is a distinct subgroup of ACC with worse outcome (1), as complete resection of the tumor is usually difficult if not impossible. In addition, the rich lymphoid drainage of the nasopharynx grants NACC a higher incidence of regional and distant metastasis. High dose radiation therapy (RT) offers the only curative opportunity for most patients with NACC. High dose of radiation is usually necessary for ACC considering its radio-resistant nature. However, the surrounding critical organs (such as brainstem and spinal cord) of nasopharynx significantly precludes escalation of RT dose that might improve disease control. Therefore, the outcome after photon-based RT is suboptimal, and the reported local control rates ranged between $35 \%$ and $65 \%(2,3)$. Because of its sharp dose distribution within the Bragg Peak region, particle beam RT can deliver higher dose to the tumor located in the nasopharynx (4). An initial study of 14 NACC patients showed that proton beam therapy (PBT) yielded a satisfactory local control rate of $79 \%$ at 5 years (5). Heavy particles (such as neutron and carbon ion) were also used to treat ACC because of their higher relative biological effects (RBE). In a randomized trial, neutron was proved to provide superior local control, as compared to photon-based RT, but with a higher incidence of treatment-induced toxicities $(6,7)$. In addition to its higher RBE, carbon-ion radiotherapy (CIRT) also has advantages in dose distribution similar to PBT, thus may further improve the therapeutic ratio over PBT. In a retrospective study on locally advanced head and neck ACC (HNACC) (8), 58 patients received intensitymodulated radiotherapy (IMRT) followed by CIRT boost, and 37 patients received IMRT only. Significantly improved 5 -year local control (59.6\% vs. 39.9\%; $\mathrm{P}=0.033)$ was observed for patients received the bimodal RT. In another series of 309 RT-naïve patients with HNACC (60\% had T4 disease), CIRT plus IMRT provided a 5-year local control rate of $58.5 \%$, and treatment-related toxicities remained moderate (9). Moreover, patients treated by CIRT plus IMRT in the 2 previous study cohorts had relatively large tumor volumes $(8,9)$. Considering the unfavorable baseline characteristics, the bimodal treatment of CIRT and IMRT provided an acceptable outcome. However, the role of CIRT exclusively for NACC has not been examined. The purpose of the current study was to evaluate the efficacy and safety of CIRT, alone or combined with PBT, for the treatment of NACC.

\section{Methods}

\section{Patients and baseline evaluations}

Between July 2016 and March 2019, 22 consecutive patients with non-metastatic nasopharyngeal adenoid cystic carcinoma (NACC) were summarized and included in the analysis. All newly diagnosed cases were confirmed by pathology, whereas either histological or radiological diagnosis was used to diagnose recurrent disease. All patients were treated by particle beam RT (CIRT or a combination of CIRT and PBT) either using a phase 2 randomized trial protocol (clinicaltrial.gov identifier: NCT02942693) or according to our institutional review board (IRB) approved standardized treatment protocol. The study was conducted in accordance with the Declaration of Helsinki (as revised in 2013) and it was approved by the institutional review board of Shanghai Proton and Heavy Ion Center (No. 200220EXP-01) with a waiver of informed consent.

Baseline evaluation included a complete medical history and physical examination, nasopharyngoscopy, lab tests (complete blood count, serum electrolytes, liver and renal function), electrocardiogram, and urinalysis. Magnetic resonance imaging (MRI) of the nasopharynx and bilateral neck was mandatory unless clinically contraindicatory. Status on distant metastasis was evaluated by whole-body positron emission tomography (PET)/computed tomography (CT) (or by chest CT, abdominal ultrasonography and bone scan). American Joint Committee on Cancer TNM staging system for nasopharyngeal cancer was used.

\section{Radiotherapy and systemic treatment}

Immobilization and set up was performed in supine position for all patients using AlphaCradle ${ }^{\circledR}$ and thermoplastic mask. Simulation CT scan with a $1.5-\mathrm{mm}$ cut of the head and neck region was conducted for all patients. MRI at treatment position was recommended and fused to simulation CT for contouring purpose. The gross tumor volume (GTV) was defined as the primary disease identified by physical examination or any imaging studies. A 5-mm margin was added to GTV to create clinical target volume (CTV). The complete nasopharynx, skull base, and bilateral upper cervical nodes were included in the CTV (lower neck was included only if positive neck nodes were 
detected). Corresponding peripheral nerves were traced (till the base of skull) and included in the CTV as well. CTV was expanded by an additional 3- to 6-mm to create planning target volume (PTV), addressing both setup error and range uncertainty. Organs at risk (OARs) delineated mainly included brainstem, spinal cord, temporal lobes, optic nerves/chiasm, lenses, cochleae, and parotid glands. And dose limits imposed on the OARs were based on normal tissue tolerance described by Emami (10). Proton and carbon-ion radiotherapy were delivered using pencil beam scanning (PBS) by the IONTRIS system (Siemens, Germany). Treatment plans were completed using multi-field optimization (MFO) with typically 2-3 fields. A combination of PBT and CIRT was used for RT-naïve patients, and CIRT alone was used for patients with recurrent disease who failed previous photonbased RT.

Two cycles of apatinib $(0.5 \mathrm{mg}$ daily, every 4 weeks as one cycle) prior to particle beam RT was prescribed to patients recruited to the phase 2 trial or when it was deemed beneficial by the attending radiation oncologists.

\section{Follow-up and toxicity evaluation}

Weekly evaluation for adverse events was performed during the entire course of RT. All patients were asked to visit their radiation oncologists 4 weeks after the completion of RT, every 3 months for the first 2 years, every 6 months till the 5 th years, and annually afterwards. MRI of the head and neck region was ordered at each visit. Thoracic CT, abdominal ultrasonography, and bone scan were performed every 6 to 12 months at the discretion of radiation oncologists.

\section{Statistics}

Overall survival (OS) time was defined as the duration from diagnosis of primary/recurrent disease until death or the last follow-up. Time to local, regional, or distant failure were calculated from diagnosis to corresponding failures. Treatment response was assessed by the Response Evaluation Criteria In Solid Tumors (RECIST) version 1.1 (11). Acute toxicities were defined as toxicities occurred within 90 days after initiation of RT, those occurred afterwards were defined as late toxicities. Toxicities were graded by the Common Terminology Criteria for Adverse Events (CTCAE) version 4.03. Survivals were calculated using Kaplan-Meier method. $\mathrm{P}$ values $<0.05$ were considered as statistically significant. All analyses were performed with $\mathrm{R}$ statistical software (version3.4.1; R Foundation, Austria).

\section{Results}

\section{Patient and treatment characteristics}

Twenty-four consecutive NACC patients were treated at SPHIC between July 2016 and March 2019. After excluding 2 patients with distant metastasis, a total of 22 patients were included in the final analysis. Among the 18 patients with newly diagnosed NACC, 11 were recruited to an institutional phase 2 randomized trial. Four patients had recurrent NACC including 2 were previously irradiated. Patients and their treatment characteristics were summarized in Table 1.

\section{Treatment response and survivals}

The median follow-up for the study cohort was 30.9 (range, 9.1-43.8) months, and all patients were alive at our last follow-up. Four and 5 patients developed local recurrence and distant metastasis, respectively. Complete remission of the tumor was observed in 11 of the 12 (91.7\%) patients who received MRI at 1 year after particle therapy. The details of treatment response were shown in Table 2. The 2-year OS, progression-free survival (PFS), local progression-free survival (LPFS), regional progression-free survival (RPFS), and distant metastasis-free survival (DMFS) rates were $100 \%$ (95\% CI, 100-100\%), 84.8\% (95\% CI, 70.2-100\%), $94.4 \%$ (95\% CI, $84.4-100 \%$ ), $100 \%$ (95\% CI, $100-100 \%$ ), and $84.8 \%$ (95\% CI, $70.2-100 \%)$, respectively (Figure 1).

\section{Acute and late toxicities}

Acute toxicities induced by particle beam radiotherapy were detailed in Table 3. Grade $\geq 3$ acute toxicities were uncommon but included mucositis $(9.1 \%)$ and xerostomia (4.5\%). Grade 1-2 late toxicities included xerostomia (12 cases, $54.5 \%$ ), hearing impairment ( 5 cases, $22.7 \%$ ), tinnitus ( 3 cases, 13.6\%), visual acuity decrease ( 3 cases, 13.6\%), cranial neuropathy ( 6 cases, $27.3 \%$ ), and temporal lobe necrosis ( 1 case, $4.5 \%$ ). No late toxicities of grade $\geq 3$ was observed.

\section{Discussion}

In this study, CIRT or CIRT in combination with PBT appeared to be an effective treatment option for NACC. As a radio-resistant malignancy, the remission of NACC after particle therapy was slow in the current study. Nevertheless, 
Table 1 Baseline and treatment characteristics*

\begin{tabular}{|c|c|}
\hline Characteristics & No. of patients (\%) \\
\hline \multicolumn{2}{|l|}{ Age (years) } \\
\hline Median [range] & 42 [21-64] \\
\hline$<60$ years & $20(90.9)$ \\
\hline$\geq 60$ years & $2(9.1)$ \\
\hline \multicolumn{2}{|l|}{ Gender } \\
\hline Female & $18(81.8)$ \\
\hline Male & $4(18.2)$ \\
\hline \multicolumn{2}{|l|}{ Tumor category } \\
\hline $\mathrm{T} 1$ & $1(4.5)$ \\
\hline $\mathrm{T} 2$ & 0 \\
\hline T3 & $2(9.1)$ \\
\hline $\mathrm{T} 4$ & $19(86.4)$ \\
\hline \multicolumn{2}{|l|}{ Node category } \\
\hline NO & $16(72.7)$ \\
\hline N1 & $3(13.6)$ \\
\hline N2 & $3(13.6)$ \\
\hline N3 & 0 \\
\hline \multicolumn{2}{|l|}{ Disease stage } \\
\hline 1 & $1(4.5)$ \\
\hline II & 0 \\
\hline III & $2(9.1)$ \\
\hline IV & $19(86.4)$ \\
\hline Median tumor volume $(\mathrm{mL})$ & $51.3(20.7-281.0)$ \\
\hline \multicolumn{2}{|l|}{ Disease status } \\
\hline Newly diagnosed & $18(81.8)$ \\
\hline Recurrent & $4(18.2)$ \\
\hline \multicolumn{2}{|l|}{ Surgery ${ }^{\dagger}$} \\
\hline Yes & $8(36.4)$ \\
\hline No & $14(63.6)$ \\
\hline \multicolumn{2}{|l|}{ Induction apatinib therapy } \\
\hline Yes & $6(27.3)$ \\
\hline No & $16(72.7)$ \\
\hline \multicolumn{2}{|l|}{ Radiotherapy modality } \\
\hline PBT + CIRT & $20(90.9)$ \\
\hline CIRT alone ${ }^{\ddagger}$ & $2(9.1)$ \\
\hline \multicolumn{2}{|l|}{ Re-irradiation } \\
\hline Treatment naïve & $20(90.9)$ \\
\hline
\end{tabular}

Table 1 (continued)
Table 1 (continued)

\begin{tabular}{lc}
\hline Characteristics & No. of patients (\%) \\
\hline Re-irradiation & $2(9.1)$ \\
Median dose to GTV (Gy) & $71.0(63.0-73.5)$ \\
\hline${ }^{*}$, percentages may not add up to $100 \%$ due to rounding; ${ }^{\dagger}$, for \\
all patients who received surgery, residual disease was detected; \\
${ }^{\ddagger}$, two patients with recurrent NACC who received previous RT \\
were treated by CIRT alone. PBT, proton beam therapy; CIRT, \\
carbon-ion radiotherapy; GTV, gross tumor volume.
\end{tabular}

all but one patients with follow-up information at 1 year after completion of treatment achieved complete remission. The response rate of NACC to CIRT alone or combined with PBT in our cohort was relatively higher, compared to IMRT plus CIRT boost reported in the previous study (9). With a median follow-up of 30.9 months, the 2-year OS rate, $\mathrm{PFS}$ rate, LPFS rate, RPFS rate and DMFS rate were $100 \%, 84.8 \%, 94.4 \%, 100 \%$, and $84.8 \%$, respectively. On the other hand, grade 3 or above acute toxicities only were observed in 3 patients, including 2 patients (9.1\%) with grade 3 mucositis and 1 patient $(4.5 \%)$ with grade 3 xerostomia. No severe late toxicities were observed. To the best of our knowledge, this is the first study exclusively reported the outcomes of NACC treated by CIRT with or without PRT. In the current study cohort, more than $95 \%$ of the patients had T3/T4 disease (86.4\% had T4 disease), and all patients had inoperable/residual disease. Therefore, this study reported the outcomes of NACC patients with unfavorable prognostic characteristics.

NACC is a special subgroup of ACC with worse outcomes due to its predilection of regional and distant metastasis. In addition to its high incidence of distant metastasis, local recurrence remains as a major cause of treatment failure due to the difficulty of achieving gross total resection of the primary disease by surgery. As such although the standard of care for NACC includes surgery, radiotherapy is nearly universally required, especially for locally advanced disease. In an early study of 26 NACC patients (12 had locally advanced disease), 22 received radiotherapy with or without surgery/chemotherapy (3). After a median follow-up of 48 months, the 2- and 5-year locoregional control rates were only $60.6 \%$ and $34.6 \%$, respectively. In another series of 36 NACC patients $(78 \%$ 
Table 2 Response to particle beam radiation therapy*

\begin{tabular}{|c|c|c|c|c|c|}
\hline Response & At completion of RT & 3 months after RT & 6 months after RT & 9 months after RT & 12 months after RT \\
\hline CR & $2(9.1)$ & $4(26.7)$ & $9(56.3)$ & $11(84.6)$ & $11(91.7)$ \\
\hline PR & $5(22.7)$ & $9(60.0)$ & $6(37.5)$ & $1(7.7)$ & 0 \\
\hline SD & $15(68.2)$ & $2(13.3)$ & $1(6.3)$ & $1(7.7)$ & $1(8.3)$ \\
\hline
\end{tabular}

*, percentages may not add up to $100 \%$ due to rounding; ${ }^{\dagger}$, number of patients with information on treatment response at corresponding time points. CR, complete response; PR, partial response; SD, stable disease.

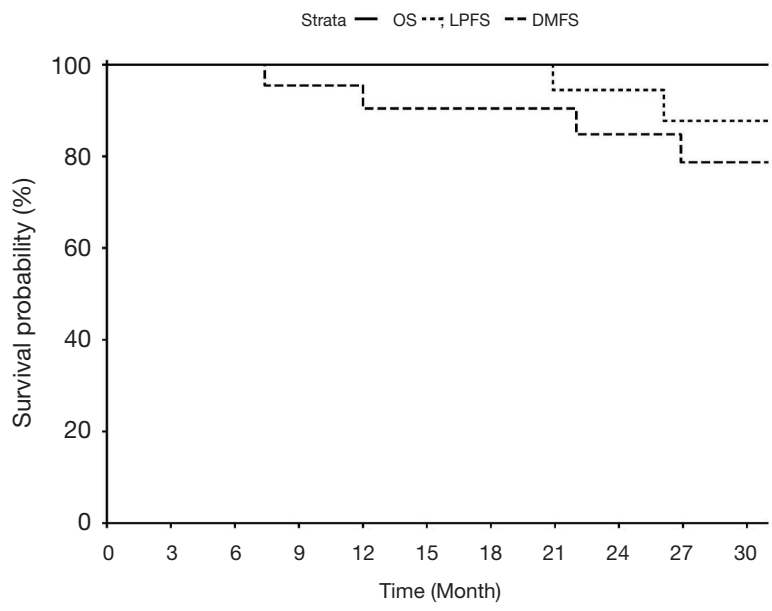

Figure 1 The survival curves of OS, LPFS and DMFS for the study cohort. OS, overall survival; LPFS, local progression-free survival; DMFS, distant metastasis-free survival.

had T3/4 disease), radiotherapy yielded an acceptable locoregional failure-free survival rate (LRFFS) of $82.5 \%$ at 2 years; however, the long-term LRFFS at 5 years was $63.4 \%$ (2). The unfavorable local control of NACC treated by photon-based radiotherapy is probably due to the resistant nature of ACC. In addition, surrounding critical organs such as brain stem, spinal cord and temporal lobes, significantly limit the radiation dose that could be delivered to the tumor volume.

PBT and CIRT can provide a more focused dose distribution because of its characteristic Bragg Peak. Thus, higher dose could be delivered to the tumor volume while sparing the surrounding normal tissues. In a series of 14 patients with locally advanced NACC, Gentile $e t$ al. showed that high dose PBT could provide satisfactory local control, despite the tumor volume of the cohort was relatively small compared to the current study (5). In that study, all patients had inoperable/residual disease, and the median dose of
PBT to CTV was 73.8 Gy (RBE). After a median followup of 69 months, the local control rate was $79 \%$. The acute toxicities were mild, and only 1 patient had grade 3 toxicity (mucositis); whereas grade 3 or above late toxicities were observed in $21 \%$ of the patients, including 1 case of grade 5 brainstem necrosis that was probably due to the high dose delivered to the surface of brainstem close to the extensive tumor.

Heavy particle beams were able to effectively eliminate resistant tumor cells owing to its higher RBE as compared to photon and proton. Neutron and carbonion radiotherapy has been previously attempted to treat HNACC. In an early randomized trial, neutron showed a significantly improved local control rate at 2 years $(67 \%$ vs. $17 \%, \mathrm{P}<0.005)$ and 10 years $(56 \%$ vs. $17 \%, \mathrm{P}=0.009)$ as compared to photon radiotherapy for patients with unresectable salivary gland tumors, though no OS benefit was detected $(6,7)$. It must be noted that neutron therapy was related to an increased incidence of severe treatmentrelated toxicities, though not fatal. In another retrospective study of 84 ACC patients of minor salivary glands (11 cases of nasopharyngeal ACC) treated by neutron in 1996 (1), NACC was found to have a significantly worse OS and local control than ACC originated from other sites. And the 5 -year OS and locoregional control rates for NACC were $31 \%$ and $0 \%$, respectively. Among the 72 patients received curative dose of neutron therapy, 10 patients ( 2 had previous full dose radiotherapy) developed grade 3/4 late toxicities. Lack of imaging guidance and advanced three-dimensional techniques was part of the reason for the unfavorable outcome for neutron therapy.

In addition to high RBE, CIRT using raster scanning technique could also provide a sharp dose distribution by depositing most of its energy in the Bragg Peak region, as PBT does (4). Jensen et al. compared the outcome of CIRT plus IMRT vs. IMRT alone in 95 patients with HNACC 
Table 3 Acute toxicities induced by particle therapy

\begin{tabular}{lcccc}
\hline Toxicities & None & Grade 1 & Grade 2 & Grade 3 \\
\hline Mucositis & $2(9.1)$ & $7(31.8)$ & $11(50.0)$ & 0 \\
Dermatitis & $9(40.9)$ & $13(59.1)$ & $1(4.5)$ & 0 \\
Xerostomia & $13(59.1)$ & $7(31.8)$ & 0 & $1(4.5)$ \\
Hearing impairment & $21(95.5)$ & $1(4.5)$ & 0 & 0 \\
Tinnitus & $21(95.5)$ & $1(4.5)$ & 0 & 0 \\
\hline
\end{tabular}

Data are expressed as $n(\%)$.

(11 patients had NACC) (8). All but one patients had locally advanced disease. The median biological equivalent dose (BED) that was delivered to the tumor was higher in the CIRT plus IMRT group than the IMRT group (76.5 vs. 66 Gy). The authors found a significantly better local control rate of CIRT plus IMRT at 5 years $(59.6 \% v s$. $39.9 \% ; \mathrm{P}=0.033$ ) as compared to IMRT alone. Although not significantly different, the number of adverse events recorded was higher in CIRT plus IMRT group, which might due to more rigorous follow-up strategy applied for this group of patients. Later in a larger series of 309 HNACC patients (5\% with NACC), the authors showed that CIRT plus IMRT yielded a 3- and 5-year local control rate of $83.7 \%$ and $58.5 \%$, respectively (9). In that study cohort, $60 \%$ of the patients had T4 disease and $21 \%$ were recurrent but RT-naïve cases. The toxicities related to CIRT plus IMRT were moderate, and severe late toxicities were uncommon. Overall, CIRT provided an acceptable outcome for patients with HNACC; however, its efficacy and safety were not reported specifically for NACC.

Distant metastasis was one of the major sources of treatment failure in our patients, which was similar to the results of previous studies $(2,3,5)$. Five of the 22 patients developed distant metastasis, and the 2-year DMFS was $84.8 \%$, substantially worse than the high LPFS of $94.4 \%$. ACC is a malignancy with high tendency of distant metastasis, and NACC patients bear higher risk for metastasis due to the rich lymphatic drainage of nasopharynx. Unfortunately, chemotherapy has not been proven efficacious for ACC thus was not used for our patients at SPHIC. Development of effective systemic treatments is urgently needed to improve the long-term outcomes for patients with ACC. In our institution, a phase 2 randomized trial is currently ongoing to examine the role of induction apatinib in the management of HNACC (NCT02942693).

A few limitations need to be addressed for our study.
Firstly, the sample size of the current cohort of patients was not sufficient to conduct prognostic analyses, albeit it is the largest study on CIRT due to the rarity of NACC. Secondly, considering the slow growing feature of ACC, a median follow-up time of 30 months is relatively short. As such, longer follow-up is necessary to examine the true efficacy of CIRT.

\section{Conclusions}

Our results demonstrated that CIRT alone or in combination with PBT is an effective and safe treatment option for NACC. The 2-year OS, PFS, LPFS, RPFS and DMFS rates were $100 \%, 84.8 \%, 94.4 \%, 100 \%$, and $84.8 \%$, respectively. Toxicities related to particle beam radiotherapy were infrequent and mild. The only observed severe acute toxicities were mucositis and xerostomia. No severe late toxicities were observed. Longer follow-up is needed to confirm the long-term benefit of particle beam radiotherapy for patients with NACC.

\section{Acknowledgments}

Funding: This study was supported by Science and Technology Commission of Shanghai Municipality (Project No. 19411951000), and Clinical Research Plan of Shanghai Shenkang Hospital Development Center (Project No. SHDC12017X13).

\section{Footnote}

Conflicts of Interest: All authors have completed the ICMJE uniform disclosure form (available at https://atm. amegroups.com/article/view/10.21037/atm-20-1767/coif). The authors have no conflicts of interest to declare.

Ethical Statement: The authors are accountable for all 
aspects of the work in ensuing that questions related to the accuracy or integrity of any part of the work are appropriately investigated and resolved. The study was conducted in accordance with the Declaration of Helsinki (as revised in 2013) and it was approved by the institutional review board of Shanghai Proton and Heavy Ion Center (No. 200220EXP-01) with a waiver of informed consent.

Open Access Statement: This is an Open Access article distributed in accordance with the Creative Commons Attribution-NonCommercial-NoDerivs 4.0 International License (CC BY-NC-ND 4.0), which permits the noncommercial replication and distribution of the article with the strict proviso that no changes or edits are made and the original work is properly cited (including links to both the formal publication through the relevant DOI and the license). See: https://creativecommons.org/licenses/by-nc-nd/4.0/.

\section{References}

1. Douglas JG, Laramore GE, Austin-Seymour M, et al. Neutron radiotherapy for adenoid cystic carcinoma of minor salivary glands. Int J Radiat Oncol Biol Phys 1996;36:87-93.

2. Cao CN, Zhang XM, Luo JW, et al. Primary salivary gland-type carcinomas of the nasopharynx: prognostic factors and outcome. Int J Oral Maxillofac Surg 2012;41:958-64.

3. Liu TR, Yang AK, Guo X, et al. Adenoid cystic carcinoma of the nasopharynx: 27-year experience. Laryngoscope 2008;118:1981-8.

Cite this article as: $\mathrm{Hu} \mathrm{J}, \mathrm{Hu} \mathrm{W}$, Gao J, Yang J, Qiu X, Huang Q, Kong L, Lu JJ. The role of carbon-ion radiotherapy in the treatment of adenoid cystic carcinoma of the nasopharynx. Ann Transl Med 2022;10(22):1198. doi: 10.21037/ atm-20-1767
4. Durante M, Orecchia R, Loeffler JS. Charged-particle therapy in cancer: clinical uses and future perspectives. Nat Rev Clin Oncol 2017;14:483-95.

5. Gentile MS, Yip D, Liebsch NJ, et al. Definitive proton beam therapy for adenoid cystic carcinoma of the nasopharynx involving the base of skull. Oral Oncol 2017;65:38-44.

6. Griffin TW, Pajak TF, Laramore GE, et al. Neutron vs photon irradiation of inoperable salivary gland tumors: results of an RTOG-MRC Cooperative Randomized Study. Int J Radiat Oncol Biol Phys 1988;15:1085-90.

7. Laramore GE, Krall JM, Griffin TW, et al. Neutron versus photon irradiation for unresectable salivary gland tumors: final report of an RTOG-MRC randomized clinical trial. Radiation Therapy Oncology Group. Medical Research Council. Int J Radiat Oncol Biol Phys 1993;27:235-40.

8. Jensen AD, Nikoghosyan AV, Poulakis $M$, et al. Combined intensity-modulated radiotherapy plus raster-scanned carbon ion boost for advanced adenoid cystic carcinoma of the head and neck results in superior locoregional control and overall survival. Cancer 2015;121:3001-9.

9. Jensen AD, Poulakis M, Nikoghosyan AV, et al. High-LET radiotherapy for adenoid cystic carcinoma of the head and neck: 15 years' experience with raster-scanned carbon ion therapy. Radiother Oncol 2016;118:272-80.

10. Emami B, Lyman J, Brown A, et al. Tolerance of normal tissue to therapeutic irradiation. Int J Radiat Oncol Biol Phys 1991;21:109-22.

11. Eisenhauer EA, Therasse P, Bogaerts J, et al. New response evaluation criteria in solid tumours: revised RECIST guideline (version 1.1). Eur J Cancer 2009;45:228-47. 